\title{
ALGEBRAIC DIVISION RING EXTENSIONS ${ }^{1}$
}

\section{CARL FAITH}

I. Introduction. A ring $A$ is radical over a subring $B$ in case some power $a^{n(a)}$ of each $a \in A$ lies in $B$. Some of the recent literature on the structure of rings has been devoted to the problem of finding general conditions on $A$ which imply commutativity of $A$ when $B$ is the center of $A$ and $A / B$ is radical (see Kaplansky [10], Herstein [5], and especially Drazin [1] where much of the previous work along these lines is summarized and extended). This problem can be generalized in at least three ways. One can seek conditions on $A$ which imply commutativity of $A$ when $A / B$ is radical and $B$ is successively: (1) an arbitrary proper subring; (2) an arbitrary commutative subring; (3) a proper division (or even simple) subring. ${ }^{2}$ The present paper includes a complete solution to the case (1). Cases (2) and (3) are fully treated in a following article [3]. In connection with (1) the main result is:

THEOREM A. If a simple ring $A$ with minimal one-sided ideals is radical over a proper subring $B$, then $A$ is a (commutative) field.

This is the best possible result in the sense that there exist noncommutative primitive rings with minimal one-sided ideals which are radical over proper (simple) subrings (see §IV). Whether or not Theorem A can be extended to arbitrary simple rings is an open problem (cf. Footnote 6 and Theorem 2). Note, however, until the existence of simple nil rings is ruled out, an affirmative solution to this problem will be beyond reach.

Theorem $\mathrm{A}$ has the following important implication for the generation of the noncommutative ring $A$ : If to each $a \in A, n(a)$ is a corresponding natural number, then $A$ is generated by the set $\left\{a^{n(a)} \mid a \in A\right\}$. This extends the result for division rings of Hua where $\{n(a) \mid a \in A\}$ is a bounded set and Kaplansky's generalization where $n\left(x^{-1} a x\right)$ $=n(a)$ for all $x, a \in A$. Theorem $\mathrm{A}$ also has the following interesting corollary (extended in [3] to arbitrary primitive rings) which can be regarded as another natural generalization of the Wedderburn, Jacobson, and Kaplansky theorems for division rings: To each noncentral element $a$ of a noncommutative simple ring $A$ containing $a$

Received by the editors July 25, 1958 and, in revised form, February 9, 1959.

1 This paper was presented to the International Congress of Mathematicians, Edinburgh, August 18, 1958.

${ }^{2}$ I wish to take this opportunity to thank Professor M. Henriksen for suggesting (3), and for several stimulating conversations regarding Theorem A below. 
minimal left ideal, there corresponds an element $b \in A$ such that no power of $b$ commutes with $a$. Corollary $1^{\star}$, $§ I V$, offers several more general results on the generation of simple algebras.

Results similar to Theorem $\mathrm{A}$ are obtained for extensions $A / B$ of the Ikeda [7], Herstein [4], and Nakayama $[11 ; 12]$ types. In fact, Theorem A is a by-product of results achieved in a far more general program described in the next paragraphs.

Let $S=S(\Phi)$ be a set of nonconstant polynomials with zero constant terms in the variable $x$ over the field $\Phi$. Such a collection of polynomials will be called an $S$-set. If $A$ is an algebra over $\Phi$, and if $B$ is a subalgebra of $A$, then $A / B$ is $S$-algebraic provided that to each $a \in A$ there exists $f_{a}(x) \in S$ such that $f_{a}(a) \in B$. Let $P(A, S, B)$ denote the proposition:

$P(A, S, B):$ If $A$ is $S$-algebraic over $B$, then $A$ is commutative. An $S$-set is defined to be:

(1.1) an $S^{\Delta}$-set if $P(A, S, B)$ is true whenever $A$ is a division algebra, and $B$ is the center of $A$;

(1.2) an $S^{\mathbf{\Lambda}}$-set if $P(A, S, B)$ is true whenever $A$ is a division algebra, and $B$ is a subalgebra $\neq A$;

(1.3) an $S^{\star}$-set if $P(A, S, B)$ is true whenever $A$ is a simple algebra with minimum condition, and $B$ is a subalgebra $\neq A$.

In $\S \S I I$ and III (also see $\S \mathrm{V}$ ), every presently known $S^{\Delta}$-set is shown to be an $S^{\mathbf{\Lambda}}$-set. In $\S \mathrm{IV}$, the $S^{\mathbf{\Lambda}}$-sets in turn, with certain restrictions on the coefficients of the polynomials (e.g., for every $\left.f(x) \in S^{\boldsymbol{\Lambda}}, f(1) \neq 0\right)$, are proved to be $S^{\star}$-sets. For the latter $S$-sets, $P(A, S, B)$ is also true when $A$ is a simple algebra with a minimal left ideal, and $B$ is a subalgebra $\neq A$. All of which leaves the haunting question: Is every $S^{\Delta}$-set an $S^{\mathbf{\Lambda}}$-set? ${ }^{3}$

II. Radical division ring extensions. In this section the division ring case of Theorem $\mathrm{A}$ is established. $D$ will always be a division ring, and $\Delta$ a subring of $D$. When $D / \Delta$ is radical, one easily verifies that $\Delta$ is necessarily a division subring (see Lemma 3 , §III, in this connection), a fact which will be used hereafter without reference. Throughout this section $Q^{\prime}$ denotes the centralizer in $D$ of any subset $Q$ of $D$; $d \in D$ is radical over $Q$ if $d^{n} \in Q$ for some $n \geqq 1$.

Lemma 1. Let $D$ be a division ring and $\Delta$ a proper division subring.

${ }^{3}$ This can be answered affirmatively under the additional hypothesis that $S^{\Delta}$ forms a semi-group under composition of polynomials. (The proof of this, a modification of the proof of Theorem B below in which polynomials play the rôle of the powers $X^{n}$, will appear in a following article, Radical extensions of rings.) This immediately raises another question: Is the semi-group generated by an $S^{\Delta}$-set also an $S^{\Delta}$-set? 
Assume that there exist elements $v, d \in D, d \in \Delta, v \notin \Delta$ such that $d$ is not radical over $v^{\prime}$. Then, at most one element in the set $\left\{(v+c)^{-1} d(v+c) \mid c\right.$ $\left.\in d^{\prime} \cap \Delta\right\}$ is radical over $\Delta$.

Proof. We actually show, for elements $v, d \in D, d \in \Delta, v \in \Delta$, that if there exist different $c_{i} \in d^{\prime} \cap \Delta$ such that $\left(v+c_{i}\right)^{-1} d\left(v+c_{i}\right), i=1$ and 2 , are both radical over $\Delta$, then necessarily $d$ is radical over $v^{\prime}$. Accordingly, assume that there exist natural numbers $k_{i}$ such that

$$
\left[\left(v+c_{i}\right)^{-1} d\left(v+c_{i}\right)\right]^{k_{i}} \in \Delta,
$$

where $d \in \Delta, v \notin \Delta, c_{i} \in d^{\prime} \cap \Delta, i=1$ and 2 , and $c_{1} \neq c_{2}$. Then,

$$
d_{i}=\left(v+c_{i}\right)^{-1} d^{n}\left(v+c_{i}\right) \in \Delta,
$$

$n=k_{1} k_{2}, i=1$ and 2 , so that

$$
v d_{i}+c_{i} d_{i}=d^{n} v+d^{n} c_{i},
$$$$
i=1 \text { and } 2 \text {. }
$$

By subtracting (2.4) from (2.3), and transposing the $c_{i} d_{i}$ to the right, one obtains

$$
v\left(d_{1}-d_{2}\right)=d^{n}\left(c_{1}-c_{2}\right)+c_{2} d_{2}-c_{1} d_{1} \in-\Delta .
$$

Now, $\left(d_{1}-d_{2}\right) \neq 0$ would imply that $v \in \Delta$, contrary to its choice. On the other hand, setting $d_{1}=d_{2}$ in (2.5) produces, since $\left(c_{1}-c_{2}\right) \in\left(d^{n}\right)^{\prime}$,

$$
0=v 0=d^{n}\left(c_{1}-c_{2}\right)-\left(c_{1}-c_{2}\right) d_{1}=\left(c_{1}-c_{2}\right)\left(d^{n}-d_{1}\right) \text {. }
$$

Then, since $\left(c_{1}-c_{2}\right) \neq 0,(2.6)$ implies that $d^{n}=d_{1}$. If $d_{1}$ is replaced by $d^{n}$ in (2.1), one sees that $\left(v+c_{1}\right) \in\left(d^{n}\right)^{\prime}$. Since also $c_{1} \in\left(d^{n}\right)^{\prime}$, clearly $v \in\left(d^{n}\right)^{\prime}$, as well. Evidently, $d^{n} \in v^{\prime}$, contrary to the agreement that $d$ is not radical over $v^{\prime}$.

Lemma 2. If $D / \Delta$ is radical, if $D \neq \Delta$, and if for every pair $v, d \in D$ satisfying $d \in \Delta, v \in \Delta$, it follows that $d$ is radical over $v^{\prime}$, then $D$ is a field.

PRoof. One first shows, for every pair $x, y \in D$, that $y$ is radical over $x^{\prime}$. If $x, y \in D, y \in \Delta, x \in \Delta$, then $y$ is radical over $x^{\prime}$ by hypothesis. Next assume both $x, y \in \Delta$, and choose $a \in D, a \in \Delta$. Let $n$ and $m$ be such that $y^{n} \in a^{\prime}$, and $\left(y^{n}\right)^{m} \in(x+a)^{\prime}$. Then $y^{k} \in x^{\prime}, k=n m$, that is, $y$ is radical over $x^{\prime}$. The case $y \notin \Delta$ is reducible to the cases just decided since $y^{n} \in \Delta$ for suitable $n$. Next we show that $D$ is commutative. ${ }^{4}$ Let $A=\Phi(x, y)$ denote the division subalgebra generated by the

4 Although this is an immediate consequence of the result just proved and Theorem 1 of Herstein's paper, Two remarks on the commutativity of rings, Canad. J. Math. vol. 7 (1955) pp. 411-412, the short proof given here makes no use of the axiom of choice ( $=$ Zorn's lemma) as required in the proof of Herstein's theorem. 
pair $x, y \in D$, where $\Phi$ is the center of $D$. By the result just proved, if $a \in A$, then $a^{n}$ commutes with both $x$ and $y$ for suitable $n$. Then $A$ is radical over its center. By Kaplansky's theorem [10], $A$ is commutative. Since $x$ and $y$ were arbitrary elements of $D, D$ is commutative too.

We are now in a position to prove the division ring case of Theorem A, namely,

THEOREM B. If $D$ is a division ring which is radical over a proper subring $\Delta$, then $D$ is a field. ${ }^{5}$

Proof. The proof requires nothing more than a brief inspection of the results of Lemmas 1 and 2 . If $D$ is noncommutative, by Lemma 2, there exist elements $v, d \in D, v \in \Delta, d \in \Delta$, such that $d$ is not radical over $v^{\prime}$. But then, Lemma 1 shows that some conjugate of $d$ is not radical over $\Delta$. This contradicts the hypothesis that $D / \Delta$ is radical. The only way out is to admit the commutativity of $D$.

Let $D$ be a noncommutative division ring with center $C$. If $a \in D$, $a \notin C$, then, since $D / a^{\prime}$ is not radical, there exists $b \in D$ such that if $\sigma$ is the inner automorphism effected by $b$, then different natural numbers $n$ determine different conjugates $a \sigma^{n}, n=0,1,2, \ldots$. This sharpens Herstein's theorem [Conjugates in division rings, Proc. Amer. Math. Soc. vol. 7 (1956) pp. 1021-1022] which states that $a$ has infinitely mady conjugates, and leads to the following result: If $B$ is any noncentral subring, then there exists an inner automorphism $\sigma$ of $D$ such that different $n$ determine different isomorphisms $\sigma^{n}$ of $B$ in $D$. It is an open question in the case $B$ is at the same time a division subring $\neq D$ whether $\sigma$ can be chosen so that the isomorphs $B \sigma^{n}$ themselves are all different, $n=0,1,2, \cdots$ \{ It is known that $D$ always contains infinitely many distinct isomorphs $x^{-1} B x$ of $B$ (cf. Theorem 3 of my article [On conjugates in division rings, Canad. J. Math. vol. 10 (1958) pp. 374-380]).\}

III. On the generality of Theorem B. If $D / \Delta$ is a proper division ring extension, any condition which implies that $D / \Delta$ is radical implies commutativity of $D$. This principle underlies the proof of Theorem 1 below.

Let $\left[\alpha_{1}, \cdots, \alpha_{r}, x\right]$ denote the subring of $\Phi[x]$ generated by $x$ and $r$ fixed nonzero elements $\alpha_{1}, \cdots, \alpha_{r}$ in the field $\Phi$, and set:

5 I wish to take this opportunity to thank Professor I. Kaplansky for reading a manuscript containing an earlier version of this theorem, and for words which encouraged. Moreover, the inspiration for this theorem was supplied by his beautiful theorem [10]. 


$$
\begin{aligned}
& N\left(\alpha_{1}, \cdots, \alpha_{r}\right) \\
& \quad=\left\{x^{n}-x^{n+1} p(x) \mid p(x) \in\left[\alpha_{1}, \cdots, \alpha_{r}, x\right], n=1,2, \cdots\right\}
\end{aligned}
$$

Polynomials of the type (3.1) have been studied in connection with commutativity of rings by Jacobson [8], Kaplansky [10], Ikeda [7], Nakayama [11], Herstein [4], and Nakayama again in a paper [12] in which the main results on commutativity of division rings in the papers just mentioned are extended.

The next lemma shows that the subalgebra $\Delta$ of Theorem 1 is really a division subalgebra if $\Delta \neq\{0\}$, a fact which is needed in the proof of Theorem 1.

Lemma 3. Let $A$ be an algebra over a field $\Phi$, with identity 1 , and let $B$ be a subalgebra of $A$ such that $A / B$ is $S$-algebraic. Then $B$ contains $b^{-1}$ together with every $b \in B$ which is regular in $A$.

Proof. Let $b \in B$ be regular in $A$, and let $g(x)=\sum_{1}^{n} \alpha_{n-i} x^{i} \in S$, $\alpha_{0} \neq 0, \alpha_{i} \in \Phi, i=0, \cdots, n-1$, be such that $u=g\left(b^{-1}\right) \in B$. Then,

$$
v=b^{n} u-\alpha_{1} b-\cdots-\alpha_{n-1} b^{n-1}=\alpha_{01} \in B,
$$

so that $\alpha_{0}^{-1} v=1 \in B$. Furthermore,

$$
w=b^{n-1} u-\alpha_{1} 1-\cdots-\alpha_{n-1} b^{n-2}=\alpha_{0} b^{-1} \in B,
$$

so that $\alpha_{0}^{-1} w=b^{-1} \in B$ as desired.

Below, $\Phi(d)$ is the division subalgebra generated by $d \in D$.

THEOREM 1. Let $D$ be a division algebra over the field $\Phi$, and let $\Delta$ be a subalgebra such that to each $d \in D$ there corresponds nonzero elements $\alpha_{1}, \cdots, \alpha_{r} \in \Phi$ (depending on $d$ ) such that for each $a \in \Phi(d)$ there exists $f_{a}(x) \in N_{d}$ satisfying $f_{a}(a) \in \Delta$, where $N_{d}=N\left(\alpha_{1}, \cdots, \alpha_{r}\right)$ is a set of the type (3.1). Then $D$ is a field. ${ }^{6}$

Proof. If $d \in D$, then $L=\Phi(d)$ is $N_{d}$-algebraic over $Z=L \cap \Delta$. If $\Delta=\{0\}$, then for each $d \in D, \Phi(d)$ is $N_{d}$-algebraic over the center of $D$. $D$ is commutative in this case by Nakayama's [12, p. 23, Satz 1$]$. Now let $\Delta \neq\{0\}$. Then, Lemma 3 shows that $\Delta$ is a division subalgebra, hence $Z=L \cap \Delta$ is a field. If $d \notin \Delta$, then $L \neq Z$, so Nakayama's

${ }^{6}$ Added in proof (November 10, 1959): A paper [A remark on the commutativity of algebraic rings, Nagoya Math. J. vol. 12 (1959) pp. 39-44] by T. Nakayama has appeared in which $S$-sets more general than those of (3.1) are shown to be $S^{\Delta}$-sets. With references to pp. 42-43 of this paper (instead of [12]) the proof below verbatim shows that these are also $S^{\boldsymbol{\Delta}}$-sets. (Since these new $S^{\Delta}$-sets are semigroups with respect to composition of polynomials, this fact can be deduced also from the result cited in Footnote 2.) 
[12, p. 22, Hilfssatz 2] applies; $L$ is either purely inseparable over $Z$, or else $L$ is algebraic over a finite field. In either case, $d$ is radical over $\Delta$. Thus, $\Delta \neq\{0\}$ implies that $D / \Delta$ is radical. Then $D$ is commutative by Theorem B.

Jacobson's [8, Theorem 8] states that every algebraic division algebra over a finite field is commutative. Expressed otherwise, every $S$-set contained in $\Phi[x]$ is an $S^{\Delta}$-set if $\Phi$ is finite. The following corollary shows that every such $S^{\Delta}$-set is an $S^{\mathbf{\Lambda}}$-set. This result is an immediate consequence of Theorem 1 , since, if $\alpha_{1}, \cdots, \alpha_{r}$ represent the nonzero elements of $\Phi$, then clearly $D / \Delta$ is $N$-algebraic, where $N=N\left(\alpha_{1}, \cdots, \alpha_{r}\right)$ is a set of the type (3.1).

CoROllary. Let $D$ be a division algebra over a finite field $\Phi$, and let $\Delta$ be a subalgebra $\neq D$ such that to each $a \in D$ there exists a nonconstant polynomial $f_{a}(x) \in \Phi[x]$ such that $f_{a}(a) \in \Delta$. Then $D$ is a field.

REMARK. Herstein's result of [6] can be applied together with Theorem B to give a proof which by-passes Theorem 1 .

IV. Simple rings. In this section $A=T_{n}$ will always denote the complete algebra of $n \times n$ matrices over $T$, where $T$ is an algebra with identity over the field $\Phi$. As in the previous sections, $D$ is a division algebra over $\Phi$. If $A=D_{n}, n>1$, is algebraic over $\Phi$, and if $\Phi$ is finite, then it is known (cf., Rosenberg and Zelinsky [13]) that each $a \in A$ satisfies an equation of the form:

$$
x^{n(a)}-x^{m(a)}=0, \quad 0<m(a)<n(a) .
$$

This is conclusive evidence that none of the theorems of $\S I I$ can be extended in toto even to simple matrix algebras. Curiously enough, these results can be extended to simple algebras with minimal onesided ideals when either of the following restrictions on the coefficients of the pertinent $S$-sets (violated in $(4.0)^{7}$ ) is observed:

$$
\begin{aligned}
& \text { If } f(x)=\sum_{1}^{n} \alpha_{i} x^{i} \in S, \text { then } \alpha_{1} \neq 0 . \\
& \text { If } f(x) \in S, \text { then } f(1)=\sum_{1}^{n} \alpha_{i} \neq 0 .
\end{aligned}
$$

For convenience, any $S$-set satisfying (4.i) will be an $S_{i}$-set, $i=1$ or 2 .

Lemma 4. Every $S_{i}^{\mathbf{\Lambda}}$-set is an $S^{\star}$-set, $i=1$ or 2 .

Proof. Let $A=D_{n}$, where $D$ is a division algebra over $\Phi$, and as-

7 The point being that the $m(a)$ of (4.0) must exceed 1 for some $a \in D_{n}$, when $n>1$, and, clearly, $f(1)=0$ whenever $f(x)$ has the form (4.0). 
sume that $A$ is $S_{i}^{\mathbf{A}}$-algebraic over a subalgebra $B \neq A, i=1$ or 2 . If $n=1$, then $A=D$ is commutative in view of the definition (1.2). The method of proof is to show that $n=1$. Assume that $n>1$.

$S_{1}^{\mathbf{\Lambda}}$ CASE. Let $u$ be any element of $A$ such that $u^{2}=0$. If $f_{u}(u) \in S_{1}^{\wedge}$ has the form (4.1) and satisfies $f_{u}(u) \in B$, then $u=\alpha_{1}^{-1} f_{u}(u) \in B$. Hence, $B$ contains all $e_{i j} d, d \in D, i \neq j$, where $\left\{e_{i j} \mid i, j=1, \cdots, n\right\}$ are matrix units of $A$. Then, clearly, $B \supseteq A=D_{n}$, a contradiction.

$S_{\mathbf{2}}^{\mathbf{A}}$ CASE. It is known (see [2, Proposition 1]) that $A$ is generated as an algebra by idempotents. It is easy to see that $B$ must contain all idempotents of $A$ if $A / B$ is $S_{2}$-algebraic. Then $B=A$ is another contradiction.

By way of summary, all known $S^{\star}$-sets are listed below:

$$
\begin{aligned}
N_{1}\left(\alpha_{1}, \cdots, \alpha_{r}\right) & =\left\{x-x^{2} p(x) \mid p(x) \in\left[\alpha_{1}, \cdots, \alpha_{r}, x\right]\right\} \\
N_{2}\left(\alpha_{1}, \cdots, \alpha_{r}\right)= & \left\{x^{n}-x^{n+1} p(x) \mid p(x)\right. \\
& \left.\in\left[\alpha_{1}, \cdots, \alpha_{r}, x\right] ; p(1) \neq 1 ; n=1,2 \cdots\right\}
\end{aligned}
$$

For the sake of emphasis (see the corollary to Theorem 1), we also list

(4.5) Any $S_{i}$-set, $i=1$ or 2 , contained in $\Phi[x]$, when $\Phi$ is finite.

Lemma 5. Let $A$ be an algebra over $\Phi$, and let $\left\{B_{\alpha} \mid \alpha \in \Lambda\right\}$ be a nonempty collection of subalgebras such that each pair of elements of $A$ is contained in some $B_{\alpha}$. Suppose that each $B_{\alpha}$ is either commutative, or not $S$-algebraic over a proper subalgebra. Then $A$ has the same property.

Proof. Assume $A / B$ is $S$-algebraic over a subalgebra $B \neq A$. Choose $a, b \in A, b \in B, a \in B$, and suppose $a, b \in B_{\alpha}$. Now $B_{\alpha}$ is a proper $S$-algebraic extension of $B \cap B_{\alpha}$. Since $B_{\alpha}$ is therefore commutative, every $b \in B$ commutes with every $a \in A, a \notin B$. But then it is easy to see that $A$ is commutative.

Proposition 1. Let $A$ be as in Lemma 5, only suppose if $R \in\left\{B_{\alpha} \mid \alpha \in \Lambda\right\}$ that $R=T_{n}, n=n(R)$, where $T=T(R)$ is either a division algebra over $\Phi$, or else $T$ is a commutative algebra over $\Phi$. Then, if $A$ is $S_{i}^{\wedge}$-algebraic over a subalgebra $B \neq A, i=1$ or 2 , then $A$ is commutative.

Proof. If $R=T_{n}$ is $S_{i}^{4}$-algebraic over a subalgebra $\neq R$, then $n=1$ by the arguments of Lemma 4 . Then, $R=T$ is either $a$ priori commutative, or commutative by the definition of an $S^{\boldsymbol{\Lambda}}$-set. Then Lemma 5 applies.

Now let $A$ be a simple algebra over $\Phi$, and suppose that $A$ has a minimal one-sided ideal. By Litoff's theorem (see $[9$, p. 90]) $A$ is 
locally a matrix algebra in the sense that any finite subset $F$ of $A$ is contained in a subalgebra of the form $D_{n}, n=n(F)$, where $D=D(F)$ is a division algebra over $\Phi$. Thus $A$ satisfies the hypotheses of Proposition 1 . Since every commutative simple ring not a zero ring is a field, this establishes the next result which implies Theorem A.

Theorem $\mathrm{A}^{\star}$. Let $A$ be a simple algebra over a field $\Phi$, and suppose that $A$ contains a minimal one-sided ideal. Then, if $A$ is $S_{i}^{\mathbf{A}}$-algebraic over a subalgebra $B \neq A, i=1$ or 2 , then $A$ is a field.

REMARK. In a similar fashion, one can prove that if the algebra $A$ of Theorem $\mathrm{A}^{\star}$ is $S^{\star}$-algebraic over a subalgebra $B \neq A$, then $A$ is a field. This fact produces the following interesting results on the generation of simple algebras.

Corollary $1^{\star}$. Let $A$ be a noncommutative simple (resp. division) algebra over $\Phi$ with a minimal left ideal, and let $S$ be any $S^{\star}$-set (resp.

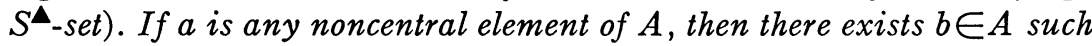
that $f(b) a \neq a f(b)$ for all $f(x) \in S$. (2) To each $a \in A$ choose $f_{a}(x) \in S$. Then $A$ is generated (as an algebra) by the set $\left\{f_{a}(a) \mid a \in A\right\}$.

Proof. (1) Since $a$ is noncentral, its centralizer $a^{\prime}$ is a proper subalgebra of $A$. Since $A$ is noncommutative, $A$ cannot be $S$-algebraic over $a^{\prime}$, that is, there exists $b \in A$ such that $f(b) \notin a^{\prime}$ for all $f(x) \in S$. (2) Clearly $A$ is $S$-algebraic over the subalgebra $B$ generated by $\left\{f_{a}(a) \mid a \in A\right\}$. Since $A$ is noncommutative, $A=B$.

An example given below shows that Theorem A cannot be extended to primitive rings, even if one assumes characteristic 0 . Let $L$ be the full ring of linear transformations (1.t.'s) in an infinite dimensional vector space $M$ over an arbitrary division ring $D$, and let $B$ (the socle) be the simple subring consisting of all $a \in L$ having finite dimensional range. Choose any $a \in L, a \notin B$, such that $a^{n} \in B$ for some $n$. For example, choose $a^{2}=0$ (I am indebted to Dr. Christine Williams Ayoub for the following remark: "Write $M=V \oplus W$, where $V$ and $W$ are isomorphic subspaces, and let $a$ map $V$ onto $W$, and $W$ onto $\{0\}$ "). Clearly the subring $A$ generated by $B$ and $a$ is radical over $B$. In fact, $b^{2} \in B$ for any $b \in A$. Since $A$ is a dense ring of 1.t.'s, $A$ is primitive (cf., e.g., $[9$, p. 175 , Structure Theorem $]$ ). This shows that Theorem A cannot be extended, in toto, to primitive rings. ${ }^{8}$ Next suppose that $D$ has characteristic 2 , and let 1 be the identity

${ }^{8}$ A partial result is contained in [3] where it is shown that if $A$ is a ring with no nil ideals $\neq\{0\}$, and if $A$ is radical over a subring $B$ which is commutative with zero Jacobson radical (respectively, $B$ a division subring $\neq A$ ), then $A$ is commutative (respectively, $A$ is a field). Cf. also the article cited in Footnote 3. 
element of $L$. Then, if $A_{1}$ is the subring generated by $B$ and 1 , $b^{2}-b \in B$ for any $b \in A_{1}$. This shows that Theorem $A^{\star}$ cannot be extended to primitive rings either, when $S_{1}^{\boldsymbol{\Lambda}}=\left\{x^{2}-x\right\}$. However, it is a fair question to ask whether Theorem A (and Theorem $\mathrm{A}^{\star}$ ) can be extended to arbitrary simple algebras. ${ }^{6}$ If $A$ is a simple algebra over a field $\Phi \neq G F(2)$, containing an idempotent $e \neq 0,1$, it is known (cf., e.g., [2]) that $A$ is generated (as an algebra) by: (1) the set of all $u \in A$ such that $u^{2}=0$; (2) the set of all idempotents in $A$. As indicated in the proof of Lemma 4 , if $A / B$ is $S_{i \text {-algebraic, }}$ then $B$ contains all elements in $(i), i=1$ or 2 . Thus, such an algebra $A$ cannot be $S_{i}$-algebraic, $i=1$ or 2 , over a subalgebra $B \neq A$, a fact which is used in the proof of the theorem below. In the statement, the term $I$-algebra is used in the sense of Jacobson [2, p. 210], namely, an algebra in which every non-nil left ideal contains a nonzero idempotent. ${ }^{9}$

TheOREM 2. Let $A$ be a simple I-algebra over a field $\Phi \neq G F(2)$, and assume that $A$ is $S_{\mathbf{A}}^{\mathbf{A}}$-algebraic, $i=1$ or 2 , over a subalgebra $B \neq A$. Then, either $A$ is a field, or $A$ is a nil algebra.

Proof. If $J$ is the Jacobson radical of $A$, then either $J=A$, or $J=\{0\}$, by the simplicity of $A$. It is well-known that $J$ contains no nonzero idempotents. Thus, either $J=A$ is nil, or $J=\{0\}$. Since $J$ contains every nil left ideal of $A, J=\{0\}$ implies that every nonzero left ideal $I$ of $A$ contains a nonzero idempotent $e(I)$. Then $A$ is a division algebra if and only if $e(I)=1$ for every such $I$. In this case, $A$ is commutative by the definition (1.2) of an $S^{\boldsymbol{\Lambda}}$-set. The case $e(I) \neq 1$ for some nonzero left ideal $I$ is ruled out by the remarks preceding Theorem 2 .

It is an open question whether there exist simple nil algebras. In proving Theorem 2, two weaker (?) questions occurred to me: (1) Does there exist a simple algebra containing a nonzero nil left ideal ${ }^{10}$ (2) Does there exist a simple algebra containing a nonzero nilpotent element all of whose algebraic elements are nilpotent?

Theorem 2 offers a criterion for deciding whether a noncommutative simple algebraic algebra (or simple $I$-algebra) $A$ over a field $\Phi \neq G F(2)$ is nil: $A$ is nil if and only if $A$ is radical over a subalgebra $B \neq A$. Then $A$ is radical over every subalgebra!

- Every algebraic algebra is an $I$-algebra [2, p. 210, Proposition 1]. An $I$-algebra is sometimes called a Zorn algebra.

${ }^{10}$ An affirmative answer would have an important application: Then, either $A$ is a simple nil ring, or else the Köthe radical of $A$ does not exist. 
V. Conclusion. Theorem 1 can be generalized to the case $D$ is a division algebra (in the sense of Jacobson $[9$, p. 16]) over a commutative integral domain $\phi$ (with identity), and $\Delta$ is a $\phi$-subalgebra, by requiring for each $d \in D$ that:

(1) the pertinent polynomials $f_{a}(x)$ in the set $N_{d}$ (defined by (3.1) in the polynomial ring $\phi[x]$ over $\phi)$ are all monic.

(2) $f_{a}(a) \in \Delta, f_{a}(x) \in N_{d}$, for each $a \in \Phi(d)$, where $\Phi$ is the field of quotients of $\phi 1$, where 1 is the identity of $D$.

The value of (1) is that the proof of Lemma 3 can be carried over verbatim to show that $\Delta$ is a $\Phi$-subalgebra of $D$ considered as an algebra over $\Phi$. (In fact, when $\Delta \neq\{0\}, \Delta$ is actually a division subring containing $\Phi$.) This reduces the situation to precisely that of Theorem 1.

TheOREM. If $A$ is a simple algebra with a minimal left ideal over a commutative integral domain $\phi$, and if there exist a subalgebra $B \neq A$ and nonzero elements $\alpha_{1}, \cdots, \alpha_{r} \in \phi$ such that to each $a \in A$ there corresponds a monic polynomial $p_{a}(x)$ in the subring of $\phi[x]$ generated by $\alpha_{1}, \cdots, \alpha_{r}$, and $x$ such that $a^{N}-a^{N+1} p_{a}(a) \in B$ for a natural number $N=N(a)$ subject to the requirement that $N(a)=1$ for all $a \in A$ if $p_{a}(1)$ $\neq 0$ for some $a \in A$, then $A$ is a field.

Proof. If $p_{a}(I)=0$ for all (idempotent) $a$, then $B$ contains all idempotents; if $N(a)=1$ for all (nilpotent) $a, B$ contains all nilpotent elements of index 2. In either case, by the proof of Lemma $4, A$ is then a division ring satisfying (1) and (2) above.

Added in proof (November 10, 1959). For a long time I thought that every $S^{\Delta}$-algebraic field extension was also a radical extension. I wish to present a counter example, and, at the same time, a new class of $S^{\Delta}$-sets, which hinge on the notion of quasi-algebraically closed (q.a.c.) fields. Here, a field $\phi$ is q.a.c. in case every finite-dimensional division algebra over $\phi$ is commutative. The q.a.c. fields include: (1) algebraically closed fields; (2) Galois fields; and (3) any field of rational functions (in a single variable) with coefficients in a field of the type (1). C. C. Tsen has proved that any field $\phi$ of type (3) is q.a.c. but the field of rational functions over (3) is not q.a.c. [Algebren über Funktionenkörpern, Göttingen dissertation, 1934]. (I am indebted to F. Kasch for this reference to Tsen's theorem.)

Clearly, any locally finite division algebra over a q.a.c. field is commutative; for example, if $D$ is an algebraic algebra over a q.a.c. field, and if $D$ is finite-dimensional over its center $C$, then $D=C$, since $D$ is evidently locally finite (cf., e.g., $[9$, p. 242 , Proposition 3 , or Theorem 1]). This leads to a new class of $S^{\Delta}$-sets. 
THEOREM. Let $\phi$ be a q.a.c. field; let $n$ be a fixed natural number; and let $S_{n}$ be any $S(\phi)$-set whose elements all have degrees $\leqq n$. Then $S_{n}$ is an $S^{\Delta}$-set.

Proof. Let $D$ be an algebra over $\phi$ such that $D / C$ is $S_{n}$-algebraic. Since $D / C$ is thereby algebraic of bounded degree, $[D: C]<\infty$ by

[8, Theorem 7]. If $D \neq C$, there exists $a \in D, a \in C$ such that the field $L=C(a)$ is separable over $C$ (cf., e.g. [8, Lemma 2]). Since $L / C$ is $S(\phi)$-algebraic, and $L \neq C$, by Herstein's theorem [6], $L$, whence $D$, is algebraic over $\phi$. Since $D / \phi$ is locally finite, as indicated above, $D=C$. Thus, $S_{n}$ is an $S^{\Delta}$-set.

Now let $\phi$ be a field (3). If $k$ is a finite algebraic field extension of $\phi$ of degree $n>1$, then to each $a \in K$ there corresponds a polynomial $f_{a}(x)$ in the polynomial ring over $\phi$ such that: (i) $f_{a}(a) \in \phi$; (ii) degree $f_{a}(x) \leqq n$; and (iii) $S_{n}=\left\{f_{a}(x) \mid a \in k\right\}$ is an $S(\phi)$-set. By the theorem, $S_{n}$ is an $S^{\Delta}$-set. If the characteristic of $\phi$ is properly chosen, say, characteristic $=0$, then one can be sure by Kaplansky's lemma [10], that $k / \phi$ is not radical. Then, $k / \phi$ is an $S^{\Delta}$-algebraic field extension which is not a radical extension. (It can be shown [see the paper cited in Footnote 4] that $S_{n}=S^{\Delta}$ is also an $S^{\mathbf{\Delta}}$-set.)

\section{REFERENCES}

1. M. P. Drazin, Rings with nil commutator ideals, Rend. Circ. Mat. Palermo vol. 6 (1957) pp. 1-14.

2. Carl Faith, Submodules of rings, Proc. Amer. Math. Soc. vol. 10 (1959) pp. 596-606.

3. - Radical extensions of rings, Proc. Amer. Math. Soc. (to appear).

4. I. N. Herstein, Structure of a certain class of rings, Amer. J. Math. vol. 75, (1953) pp. 864-871.

5. —, A theorem on rings, Canad. J. Math. vol. 5 (1953) pp. 238-241. 203.

6. —_ A theorem concerning three fields, Canad. J. Math. vol. 7 (1955) pp. 202-

7. M. Ikeda, On a theorem of Kaplansky, Osaka Math. J. vol. 4 (1952) pp. 235-240.

8. N. Jacobson, Structure theory for algebraic algebras of bounded degree, Ann. of Math. vol. 46 (1947) pp. 695-707.

9. - Structure of rings, Amer. Math. Soc. Colloquium Publications, vol. 37, Providence, 1956.

10. I. Kaplansky, $A$ theorem on division rings, Canad. J. Math. vol. 3 (1951) pp. 290-292.

11. T. Nakayama, The commutativity of division rings, Canad. J. Math. vol. 5 (1953) pp. 242-244.

12. - Über die Kommutativität gewisser Ringe, Abh. Math. Semi. Univ. Hamburg. vol. 20 (1955) pp. 20-27.

13. A. Rosenberg and D. Zelinsky, On Nakayama's extension of the $x^{n(x)}$ theorems, Proc. Amer. Math. Soc. vol. 5 (1954) pp. 484-486.

The Pennsylvania State University 